\title{
Monodispersed $\beta$-Glycerophosphate-Decorated Bioactive Glass Nanoparticles Reinforce Osteogenic Differentiation of Adipose Stem Cells and Bone Regeneration In Vivo
}

\author{
Yi Guo, Yumeng Xue, Juan Ge, and Bo Lei*
}

Design and development of highly bioactive nanoscale biomaterials with enhanced osteogenic differentiation on adipose stem cells is rather important for bone regeneration and attracting much attention. Herein, monodispersed glycerophosphate-decorated bioactive glass nanoparticles (BGN@GP) are designed and their effect is investigated on the osteogenic differentiation of adipose mesenchymal stem cells (ADMSCs) and in vivo bone regeneration. The surface-modified BGN@GP can be efficiently taken by ADMSCs and shows negligible cytotoxicity. The in vitro results reveal that BGN@GP significantly enhances the alkaline phosphatase activity and calcium biominerialization of ADMSCs either under normal or osteoinductive medium as compared to BGNs. Further studies find that the osteogenic genes and proteins including Runx2 and Bsp in ADMSCs are significantly improved by BGN@GP even under normal culture medium. The in vivo animal experiment confirms that BGN@GP significantly promotes the new bone formation in a rat skull defect model. This study suggests that bioactive small molecule decorating is an efficient strategy to improve the osteogenesis capacity of inorganic ceramics nanomaterials.

\section{Introduction}

As the burgeoning and ageing population, an increasing number of patients are suffering from bone defects caused by physical trauma, tumor, and other bone diseases. ${ }^{[1]}$ Therefore, bone tissue repair and regeneration have made considerable strides in the modern times. However, these problems are not adequately overcome by current remedies such as autografts, allografts, or synthetic bone graft substitutes. ${ }^{[2,3]}$ Although autografts are still thought as "gold standard" for bone tissue repair, the major shortcomings of autografts are the difficulties in the harvesting procedure, equivalently the donor site morbidity and the limited bone amount that can be collected. ${ }^{[4]}$ What is more, allografts suffer from the difficult balance between immune rejection and immunosuppression and virus infection. To repair and regenerate bone tissue, the

Dr. Y. Guo, Dr. Y. Xue, Dr. J. Ge, Prof. B. Lei

Frontier Institute of Science and Technology

Xi'an Jiaotong University

Xi'an 710054, China

E-mail: rayboo@xjtu.edu.cn

Dr. Y. Guo

Department of Biologic and Materials Sciences

University of Michigan

Ann Arbor, MI 48109, USA

Prof. B. Lei

National and Local Joint Engineering Research Center of Biodiagnosis and Biotherapy

The Second Affiliated Hospital of Xi'an Jiaotong University

Xi'an 710004, China

Prof. B. Lei

Instrument Analysis Center

Xi'an Jiaotong University

Xi'an 710054, China

Prof. B. Lei

Key Laboratory of Shaanxi Province for Craniofacial Precision

Medicine Research

College of Stomatology

Xi'an Jiaotong University

Xi'an 710000, China

The ORCID identification number(s) for the author(s) of this article can be found under https://doi.org/10.1002/ppsc.201900462.

DOI: $10.1002 / p p s c .201900462$ employment of stem cells and biomaterials is being investigated and has become one of the best choices in bone tissue engineering. ${ }^{[5-8]}$

As an important cell type, mesenchymal stem cells (MSCs) have been used in tissue engineering. ${ }^{[9,10]}$ MSCs have attracted much attention due to their unique capabilities of self-renewal in an undifferentiated state for prolonged time and multilineage differentiation after proper stimulation. ${ }^{[1]}$ The differentiation and the fate of MSCs are known to be governed and regulated by a variety of biological and physiochemical cues in vivo. ${ }^{[12]}$ To induce different lineage commitment, cells may require the appropriate extracellular signals to trigger or to promote this process. The biological growth factors have been widely demonstrated to induce the differentiation of MSCs. For example, bone morphogenetic proteins (BMPs), ${ }^{[13]}$ such as BMP-2 and BMP-7, are known as the most potent growth factors for directing the osteogenesis of cells like MSCs and bone formation transforming growth factors (TGF- $\beta 1$ and TGF- $\beta 3$ ) can be utilized to enhance the direct differentiation of MSCs. ${ }^{[14,15]}$ Additionally, many chemicals are frequently used in the specific osteogenic differentiation of cells in vitro. For instance, dexamethasone (DXM), ascorbic acid, and $\beta$-glycerophosphate are the typical osteogenic inducers for MSCs. ${ }^{[16]}$

Bioactive nanomaterials have attracted broad attention in bone tissue engineering studies due to their similarities to the 
nanostructured nature of extracellular microenvironment and native bone tissue. ${ }^{[17-20]}$ Bioactive nanomaterials with special morphology and size have shown positive effect on the behavior of cells and biological molecules, ${ }^{[21,22]}$ which is different from conventional bulk biomaterials. ${ }^{[23]}$ For example, nanoscale bioactive ceramics biomaterials exhibited the enhanced osteogenic differentiation on osteoblasts and stem cells. ${ }^{[24,25]}$ Relative to other bioactive ceramics and polymers, bioactive glass-based biomaterials possess good biodegradation, bonebonding, osteogenesis and angiogenesis capacity, ${ }^{[8,26,27]}$ due to their bioactive elements, and amorphous structure. Bioactive glass materials for medical devices in dentistry and bone defect repair have been approved by Food and Drug Administration, suggesting their good safety and clinical effectiveness. ${ }^{[28]}$ Compared with traditional nanostructure bioactive particles, monodispersed nanoparticles could efficiently enter into the targeted cells and regulate the cell behavior through the action on the subcellular structure..$^{[9,29,30]}$ Previous studies have shown that monodispersed bioactive glass nanoparticles (BGNs) presented enhanced cell uptake and biodegradation, and improved biominerialization ability, demonstrated promising biomedical applications in bioimaging, gene delivery, bone regeneration, and wound healing. ${ }^{[10,21,25,27,30]}$ Monodispersed BGNs and nanoscale silicon-based biomaterials could also efficiently enhance the osteogenic differentiation of osteoblasts and bone marrow stem cells. ${ }^{[31-33]}$ Recent years, our group further found that monodispersed BGNs could induce the osteogenic differentiation of adipose mesenchymal stem cells (ADMSCs) through activating the TGF-beta signaling pathway, and the molybdenum-based branched BGNs could also promote the osteogenic differentiation of ADMSCs. ${ }^{[34,35]}$ However, the monodispersed BGNs without surface modification are easy to be aggregated during the using process. In addition, the further improvement on osteogenic activity of monodispersed BGNs in vitro and in vivo is still needed.

As an important element in the osteogenic differentiation system, $\beta$-glycerophosphate (GP) is often used in different biomaterials scaffolds for bone tissue regeneration. ${ }^{[36]}$ GP was also used to modify the physical and chemical property of biomaterials for cancer therapy. ${ }^{[37]}$ In our previous study, it was shown that the abundant calcium components make BGN with great activity and exhibit strong affinity to different anions such as acetate and phosphate groups. ${ }^{[14]}$ Our recent study also demonstrated that GP could also stabilize the BGN through the strong interaction between $\mathrm{Ca}^{2+}$ in BGN and phosphate group in GP. ${ }^{[14]}$ However, the effect of GP modification on the osteogenic differentiation and bone regeneration of $\mathrm{BGN}$ is still not clear. Therefore, herein, we aim to investigate the interaction between BGN@GP and ADMSCs, and their effect on the osteogenic differentiation of ADMSCs, as well as the in vivo bone regeneration.

\section{Experimental Section}

\subsection{Preparation and Characterizations of BGN@GP Nanoparticle}

The monodispersed BGNs were synthesized using the sol-geltemplate method, according to previous report.[27] BGN@GP nanoparticles were formed through the surface complex process under mild condition (Scheme 1). Briefly, BGNs were first dispersed and sonicated in distilled (DI) water; GP was added to the BGNs solution and the mixture was sonicated again. The obtained mixture was separated by centrifugation and washed

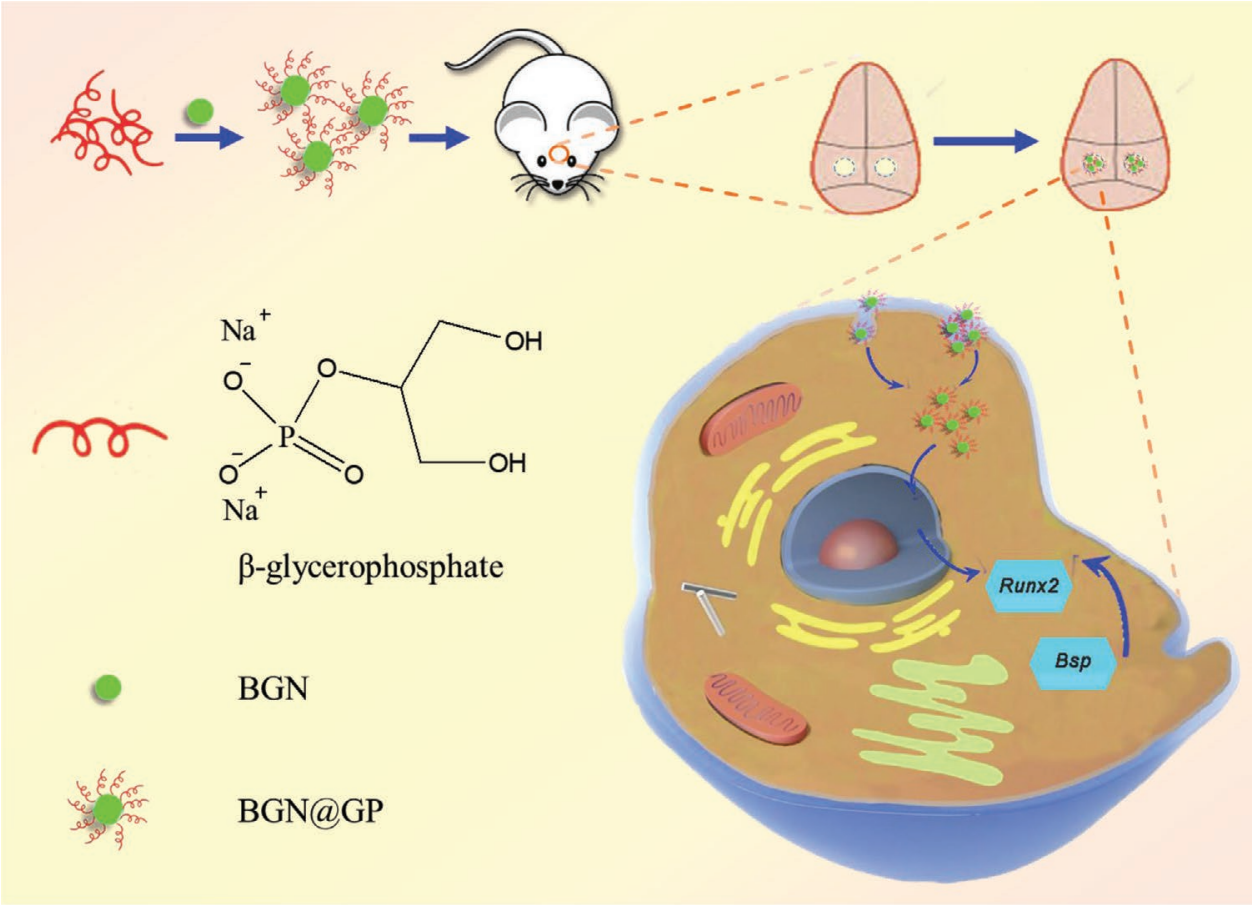

Scheme 1. Monodispersed $\beta$-glycerophosphate-decorative bioactive glass nanoparticles improve osteogenic differentiation of adipose stem cells and bone tissue regeneration in vitro and in vivo. 
with DI water to remove the redundant GP. The final product of GP-modified BGNs was named as BGN@GP. The physicochemical structure of BGN@GP nanoparticles was characterized by the Fourier-transform infrared spectroscopy (FT-IR) spectra (Nicolet 6700, Thermo Scientific Instrument), transmission electron microscopy (TEM, H-8000, Hitachi), energy dispersive spectroscopy (EDS), field emission scanning electron microscope (SU8010, Hitachi). The detailed processes are available in Supporting Information.

\subsection{Adipose Mesenchymal Stem Cells Culture and Cytotoxicity Evaluation}

ADMSCs were obtained from American Type Culture Collection. The cells were cultured in (normal growth medium) Dulbecco's Modified Eagle Medium (GIBCO) with 15\% v/v fetal bovine serum (Invitrogen), $100 \mathrm{U} \mathrm{mL}^{-1}$ penicillin, and $100 \mathrm{mg} \mathrm{mL}^{-1}$ streptomycin at $37{ }^{\circ} \mathrm{C}$ in humidified air containing $5 \% \mathrm{CO}_{2}$. The ADMSCs were seeded onto plates with normal growth medium, and the medium was changed every two days. The osteoinductive medium was made up of normal growth medium, $10 \mathrm{~nm}$ DXM, $10 \mathrm{~mm}$ b-glycerophosphate, and $50 \mu \mathrm{g} \mathrm{mL} \mathrm{m}^{-1}$-ascorbic acid. The ADMSCs viability after incubation with BGN@GP with different concentrations $\left(60-240 \mu \mathrm{g} \mathrm{mL}^{-1}\right)$ for 5 days was evaluated through the Alamar blue assay (Invitrogen) and LiveDead staining kit (Invitrogen). The fluorescent intensity of mixed medium was measured at a 570/600 nm filter by a SpectraMax fluorescence microplate reader (Molecular Devices). At least five species per sample were tested. The cells were observed by an inverted fluorescence microscope (IX53, Olympus). The Supporting Information shows the testing procedure.

\subsection{Osteogenic Differentiation Assays of ADMSCs}

The osteogenic differentiation of ADMSCs after culturing with BGN@GP at different concentrations $\left(0-80 \mu \mathrm{g} \mathrm{mL} \mathrm{m}^{-1}\right)$ was assessed by alkaline phosphatase (ALP) activity, calcium deposition analysis, osteogenic genes expressions, and osteogenic proteins. The ALP activity was determined for early culture period marker and measured on the 3rd, 7th, and 14th day. The cellular mineralization after cultivation for 3 weeks was evaluated by Alizarin Red S (ARS) kit (Genmed, Quebec). The expressions of specific osteogenic genes including Runx2 and Bsp were measured by the quantitative real-time polymerase chain reaction (Applied Biosystems 7500). The primers are shown in Table S1, Supporting Information. The RUNX2 and BSP proteins were evaluated using the immunofluoresencent staining based on the primary and secondary antibodies (Thermal). The detailed procedures for the osteogenic differentiation were similar with previous reports, as shown in the Supporting Information.

\subsection{Animal Experiment and Bone Formation Evaluation In Vivo}

The female Sprague Dawley (SD) mice used were maintained under standard animal housing conditions. SD mice were divided into two groups randomly. Animals were anaesthetized with $2 \%$ inhalation of isoflurane. A trephine was used to create two $3 \mathrm{~mm}$ craniotomy defects on the calvarial bone, and the wounds were copiously irrigated with normal saline while drilling. The calvarial disk was removed carefully to avoid injury to the underlying dura or brain. BGN@GP nanoparticles with F127 hydrogel were injectable into the bone defect, and the blank group was F127 hydrogel without nanoparticles. After 8 and 12 weeks, the calvarial bones were embedded in $4 \%$ paraformaldehyde solution, and the region of the calvarial bone was scanned with micro-CT (Y.CHEETAH*, YXLON). For examination of new bone formation, the calvarias were fixed with $10 \%$ formalin, infiltrated by resin, and hard tissue slices were observed under light microscope after Hematoxylin-Eosin (H.E.) and Masson staining. The staining process of H.E and Masson can be seen in the Supporting Information.

\subsection{Statistical Analysis}

At least three independent experiments were executed and the data were represented as average values and standard deviations (SDs). The Student- $t$ test was used to determine the statistical significance between two groups and the differences were considered as significant when $* P<0.05$ or $* * P<0.01$.

\section{Results and Discussion}

\subsection{Preparation and Characterizations of BGN@GP}

The as-prepared BGN@GP were monodisperse and spherical in shape with the size of $300-400 \mathrm{~nm}$ (Figure 1A). EDS confirmed that the main elements of BGNs were $\mathrm{Si}$ and $\mathrm{Ca}$. Comparing with BGNs,BGN@GP has more P element, suggesting the successful modification by $\beta$-glycerophosphate (Figure $1 \mathrm{~B})$. What is more, the FT-IR spectrum indicated that the characteristic bands of $\mathrm{Si}-\mathrm{O}-\mathrm{Si}$ at $1000-1100 \mathrm{~cm}^{-1}$ and the typical peaks of $\mathrm{P}-\mathrm{O}$ at 550 and $950 \mathrm{~cm}^{-1}$ (Figure $1 \mathrm{C}$ ). The amorphous structure of BGN@GP was confirmed by the X-ray diffraction (XRD) analysis (Figure 1D). These results showed that the surface modification of GP did not affect the morphology, size, and structure of BGNs (Figure S1, Supporting Information). The calculated weight ratio of GP on BGN through the thermogravimetric analysis was about $3.2 \mathrm{wt} \%$.

\subsection{ADMSCs Cytotoxicity and Proliferation Analysis}

In order to investigate the effect of BGN@GP on the growth of ADMSCs, the Alamar blue and live/dead staining assay were performed (Figure 2). It is obvious that most of the cells are alive (green), and few are dead (red), and all the ADMSCs exhibited a normal morphology after incubated with nanoparticles at $60-240 \mu \mathrm{g} \mathrm{mL}^{-1}$ (Figure $2 \mathrm{~A}$ ). The cell viability was related to the concentration of BGN@GP, ADMSCs did not show any significant death after incubation with BGN@GP for 5 days at predetermined concentrations $(0,60,120$, and $240 \mu \mathrm{g} \mathrm{mL}^{-1}$; Figure 2B). The high concentration of BGN@GP 
A

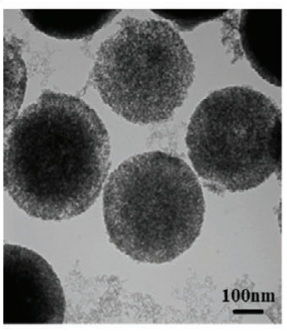

C

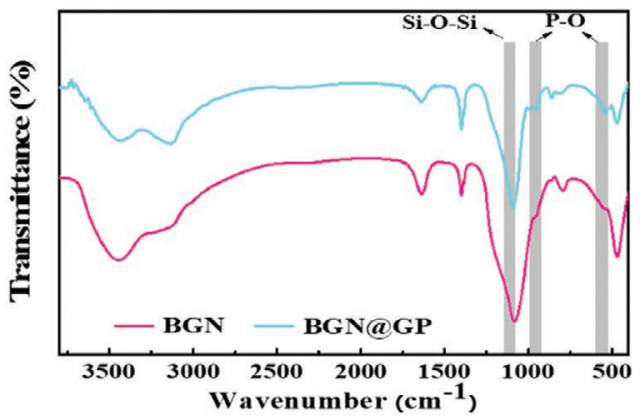

B
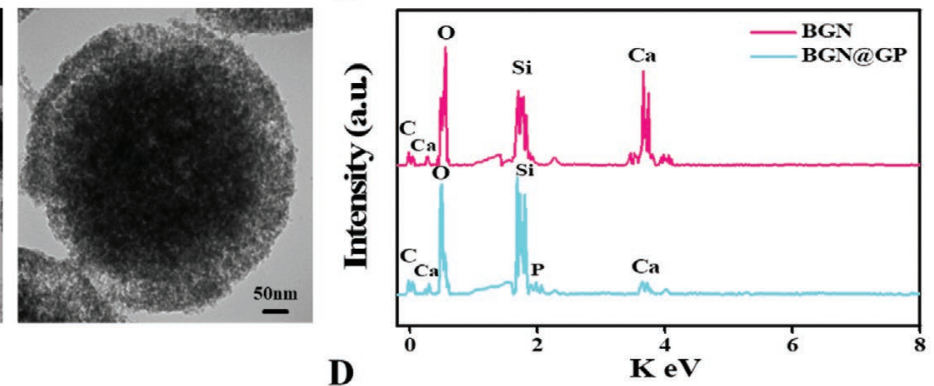

D

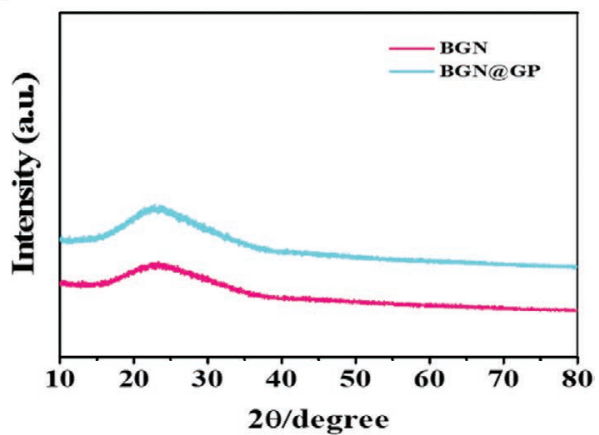

Figure 1. Morphology and structure characterization of BGN@GP. A) TEM images, B) EDS spectra, C) FT-IR spectra, and D) XRD patterns.

nanoparticles $\left(240 \mu \mathrm{g} \mathrm{mL} \mathrm{m}^{-1}\right)$ exhibited some cytotoxicity, which was probably related with the high cell uptake in ADMSCs (Figure S2, Supporting Information). In addition, at the low concentration ( $60 \mu \mathrm{g} \mathrm{mL} \mathrm{mL}^{-1}$ ), as compared with BGNs group, the BGN@GP group showed the similar cell viability and proliferation at the same concentration (Figure S3, Supporting Information). Therefore, less than $100 \mu \mathrm{g} \mathrm{mL} \mathrm{L}^{-1}$ concentration was used in subsequent experiments. These results demonstrated that low concentration BGN@GP possessed good cellular biocompatibility and may be promising for further osteogenic differentiation for ADMSCs.

\subsection{Osteogenic Differentiation Investigations of ADMSCs}

To demonstrate the effect of BGN@GP for osteogenic differentiation of ADMSCs, ALP activity, mineralization, the expression of osteogenic marker genes and proteins were measured. ADMSCs were cultured with BGN@GP in normal and osteoinductive medium. First, ALP activity was detected as the early key event during the osteogenesis. As shown in Figure 3, on the 7th day, ALP activity achieved a peak under two culture conditions. Specially, the ALP activity of the $20 \mu \mathrm{g} \mathrm{mL} \mathrm{L}^{-1}$ concentration groups were significantly stronger
$\mathbf{A}$

$0 \mu \mathrm{g} / \mathrm{mL}$

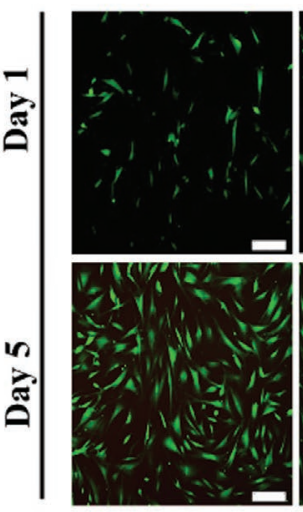

$60 \mu \mathrm{g} / \mathrm{mL}$

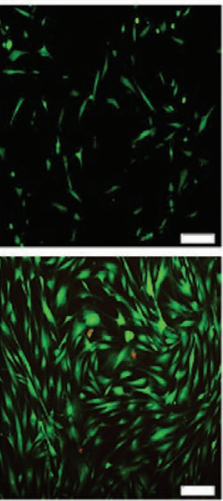

$120 \mu \mathrm{g} / \mathrm{mL}$

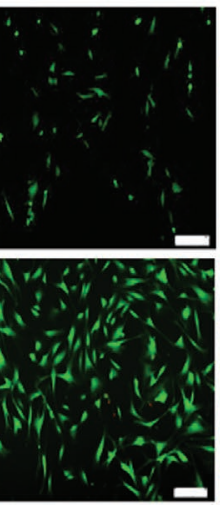

$240 \mu \mathrm{g} / \mathrm{mL}$

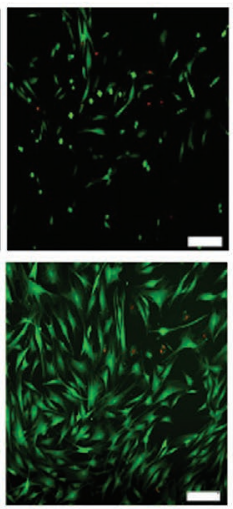

B

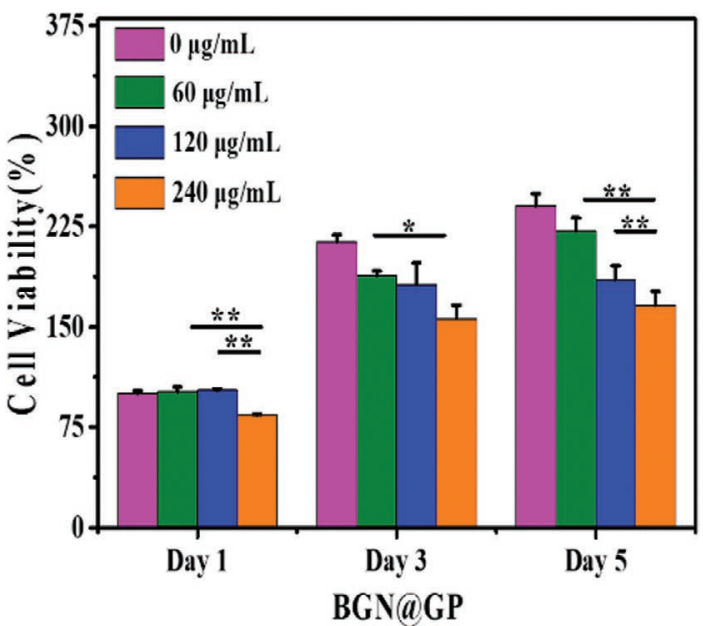

Figure 2. Cell viability and live cell imaging evaluation of BGN@GP, using the concentration of $0 \mu \mathrm{g} \mathrm{mL}^{-1}$ as a blank control. A) Live/dead staining fluorescent images of ADMSCs after incubation with BGN@GP at different concentrations $\left(0,60,120\right.$, and $240 \mu \mathrm{g} \mathrm{mL} \mathrm{L}^{-1}, \mathrm{Scale}$ bar $\left.=200 \mu \mathrm{m}\right)$. B) ADMSCs viability and proliferation after culture for 1,3 , and 5 days. $* P<0.05$ and $* * P<0.01$. 
A

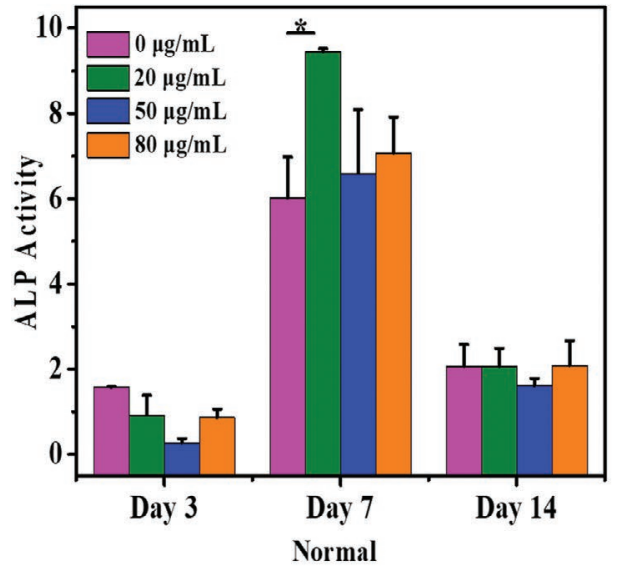

B

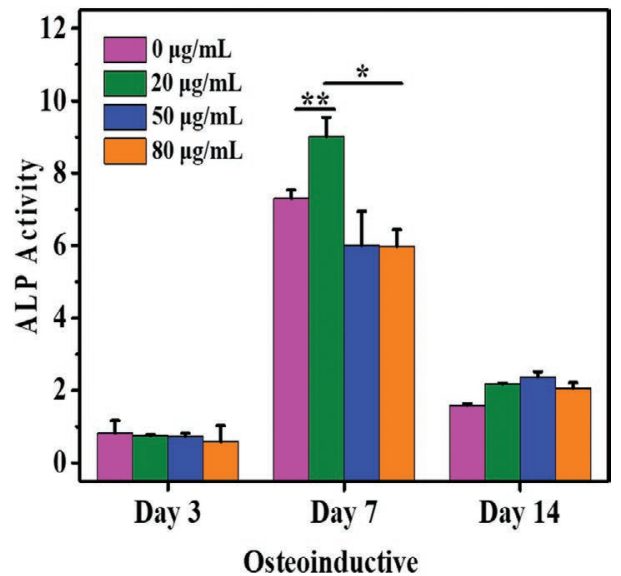

Figure 3. In vitro ALP activity evaluation of ADMSCs following induction by various concentration of BGN@GP in normal and osteoinductive medium. A) ALP activity analysis in normal growth medium. B) ALP activity in osteoinductive medium. The $0 \mu \mathrm{g} \mathrm{mL}^{-1} \mathrm{concentration}$ group as control, $* P<0.05$ and $* * P<0.01$.

than the blank groups (Figure 3A). In the meantime, the same tendency occurred in osteoinductive medium groups, the ALP activity of the $20 \mu \mathrm{g} \mathrm{mL} \mathrm{m}^{-1}$ concentration BGN@GP groups were the highest among all groups (Figure 3B).Comparing with the same concentration of BGNs $\left(20 \mu \mathrm{g} \mathrm{mL} L^{-1}\right)$, the ALP activity of BGN@GP groups were significantly enhanced than BGNs in both normal and osteoinductive groups (Figure S4, Supporting Information). On the 14th day, ALP activity showed

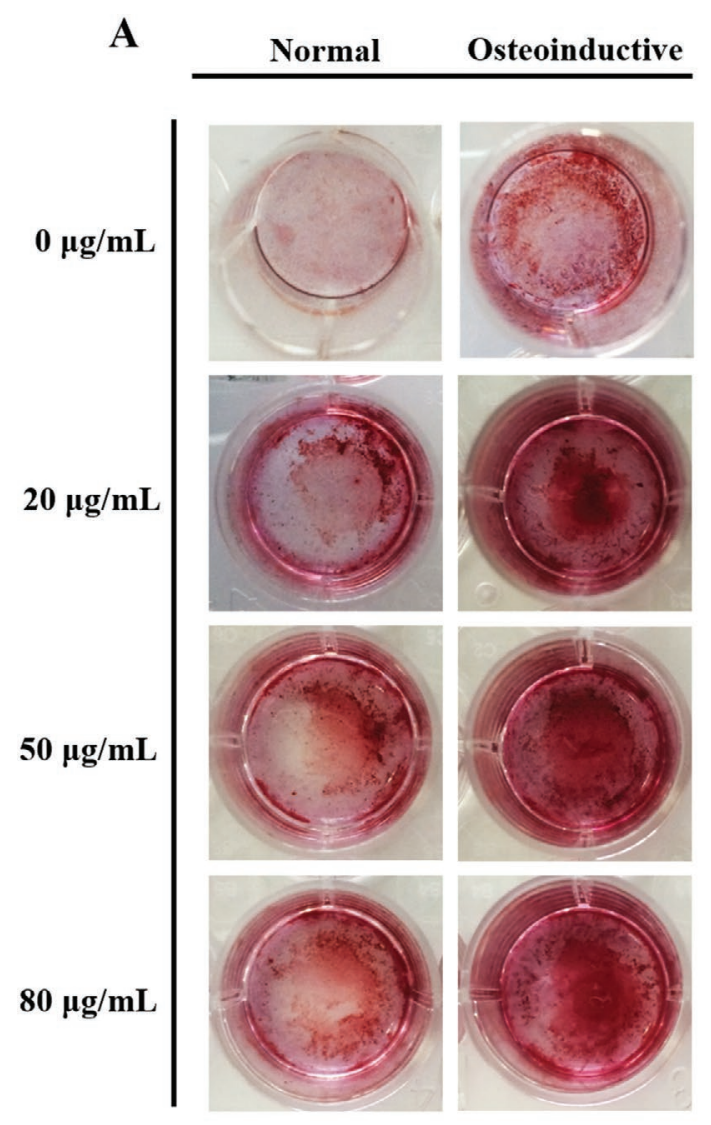

B
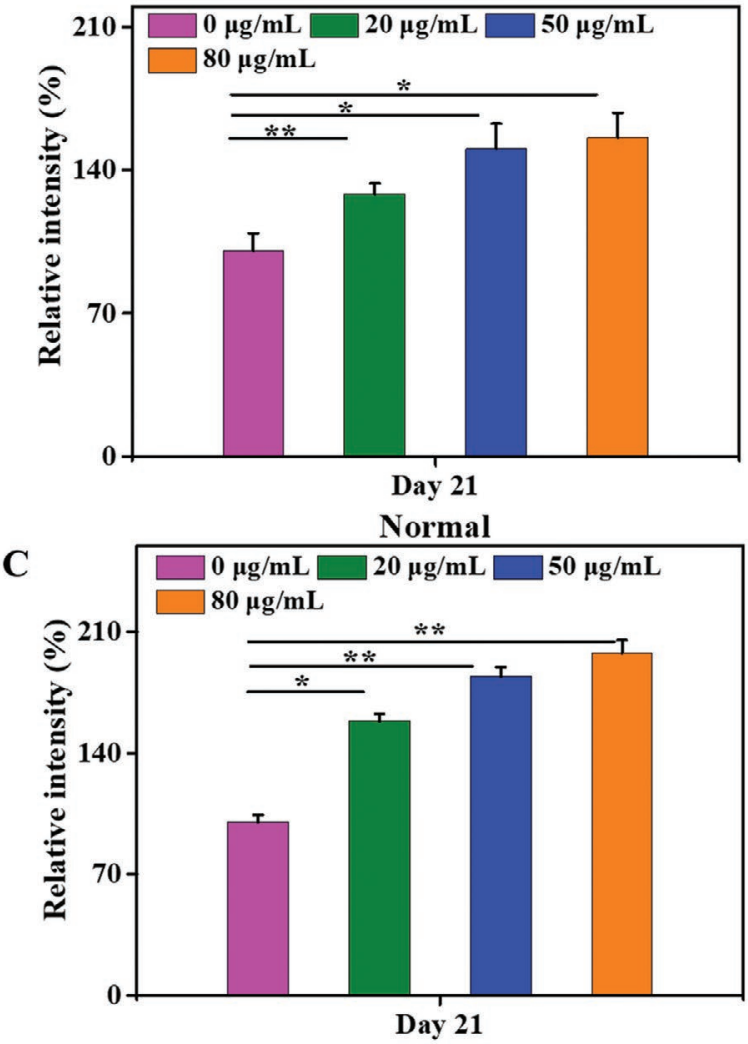

Osteoinductive

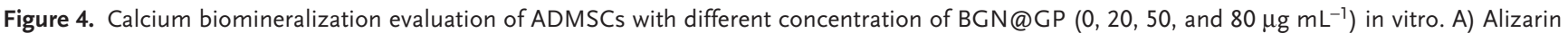
red staining assay at 21 days. $B, C)$ Relative gray level analyzed by image / based on the alizarin red staining images. The concentration of $0 \mu \mathrm{mg} \mathrm{L}^{-1}$ was regarded as a blank control. $* P<0.05$ and $* * P<0.01$. 
A

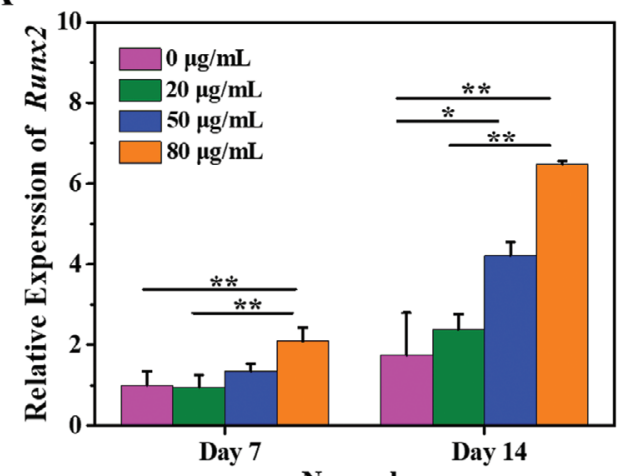

C

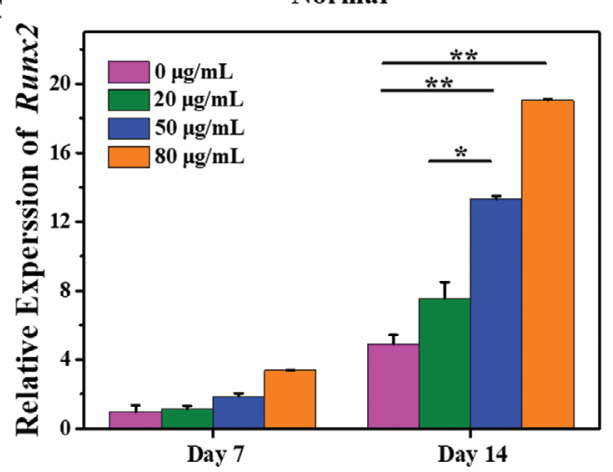

Osteoinductive
B

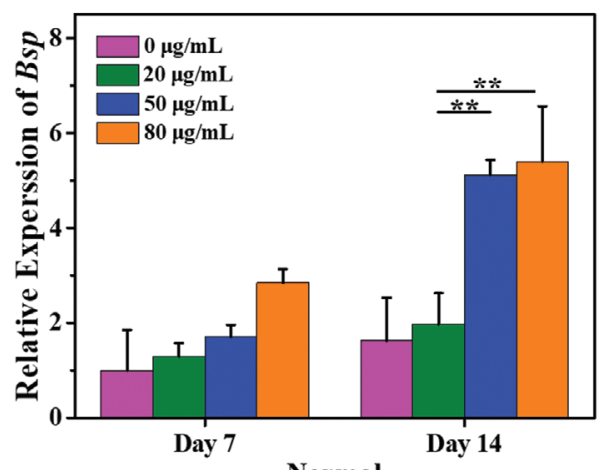

D

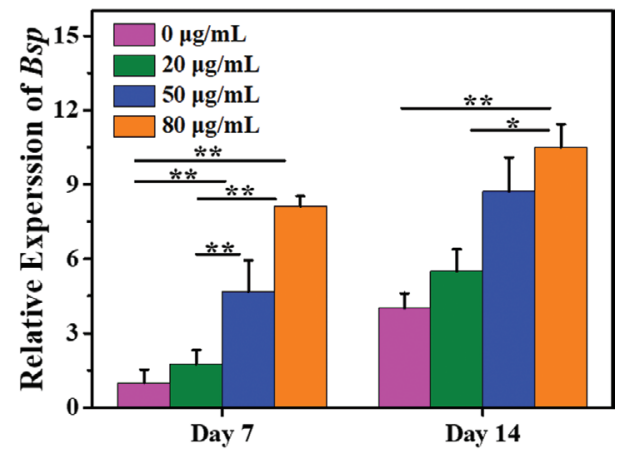

Osteoinductive

Figure 5. Relative expression of bone-specific marker genes of ADMSCs cultured in normal and osteoinductive medium with different concentration of BGN@GP, during 7 and 14 days. A,B) The relative expression of Runx2 and Bsp with normal growth medium. C,D) The relative expression of Runx2 and Bsp with osteoinductive medium. The expression of these genes was normalized against the housekeeping gene Gapdh and calculated by the $\Delta \triangle C T$ method. $* P<0.05$ and $* * P<0.01$.

no significant difference among these groups. These results indicated that the ALP activity of ADMSCs was significantly enhanced by the GP decorating on BGNs.

Calcium deposition was evaluated by ARS staining (Figure 4). Dark red area indicated the presence of calcium phosphate deposition in cellular matrix. After 21 days, the ARS staining photographs showed that BGN@GP groups had more obvious dark red areas than the blank groups, especially for the $80 \mu \mathrm{g} \mathrm{mL} \mathrm{m}^{-1}$ concentration BGN@GP groups (Figure 4A). The gray level analysis presented that the $80 \mu \mathrm{g} \mathrm{m}^{-1} \mathrm{BGN} @$ GP groups showed higher calcium deposition (Figure 4B,C). In both normal and osteoinductive condition, the ARS relative intensity of BGN@GP groups was significantly improved than BGNs groups under the $80 \mu \mathrm{g} \mathrm{mL}^{-1}$ concentration (Figure S5, Supporting Information). These results indicated that BGN@ GP had a promotive effect on the mineralization of ADMSCs, even significantly stronger than the effect of BGNs.

In order to better understand the osteogenesis, the gene expression profile of the ADMSCs cultured with BGN@GP was performed (Figure 5). After 7 and 14 days, the relative Runx2 (early-stage marker) expression of ADMSCs in $80 \mu \mathrm{g} \mathrm{mL}-1$ concentration BGN@GP groups was higher than the other groups (Figure 5A,B). For Bsp (late-stage marker) expression, the 50 and $80 \mu \mathrm{g} \mathrm{mL} \mathrm{L}^{-1}$ concentration of BGN@GP groups expressed similarly, which showed higher expression level than $20 \mu \mathrm{g} \mathrm{mL}^{-1}$ BGN@GP groups and the blank groups under normal and osteoinduction conditions (Figure 5C,D). We also detected the Runx2 and Bsp expression of BGNs at $80 \mu \mathrm{g} \mathrm{mL}^{-1}$ concentration. Comparing with BGN@GP, the expression level in BGNs group was significantly lower on the 14th day with different conditions (Figure S6, Supporting Information).

After cultured for 21 days, RUNX2 and BSP proteins expression were detected by immunofluorescent staining (Figure 6). For RUNX2 protein, the $80 \mu \mathrm{g} \mathrm{mL} \mathrm{L}^{-1}$ BGN@GP groups which had the strongest positive staining than other groups under osteoinductive medium. Among groups with normal medium, dispersive green fluorescence could be seen in $80 \mu \mathrm{g} \mathrm{mL} \mathrm{m}^{-1}$ BGN@GP groups, and less green staining could be seen in other concentration groups (Figure 6A). For BSP protein, only the 50 and $80 \mu \mathrm{g} \mathrm{m}^{-1}$ BGN@GP groups had slightly green fluorescence in normal condition. However, under osteoinductive medium, the BSP protein expression was significantly enhanced. The green area and intensity of 50 and $80 \mu \mathrm{g} \mathrm{mL}^{-1} \mathrm{BGN} @ \mathrm{GP}$ groups were higher than the blank groups, and the green fluorescence of $20 \mu \mathrm{g} \mathrm{mL}^{-1}$ BGN@ GP groups was weakest (Figure 6B). Moreover, the images of immunofluorescent staining were used to gray level analysis by image J software. We found that the results of gray level analysis were consistent with immunofluorescent images results (Figure S7, Supporting Information). These data indicated that BGN@GP can significantly improve osteogenic differentiation in vitro. 
A

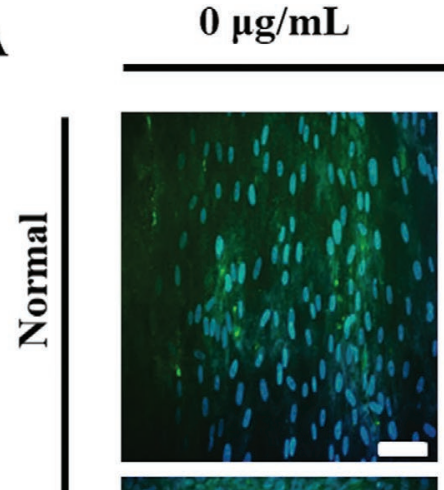

$20 \mu \mathrm{g} / \mathrm{mL}$

$50 \mu \mathrm{g} / \mathrm{mL}$

$80 \mu \mathrm{g} / \mathrm{mL}$
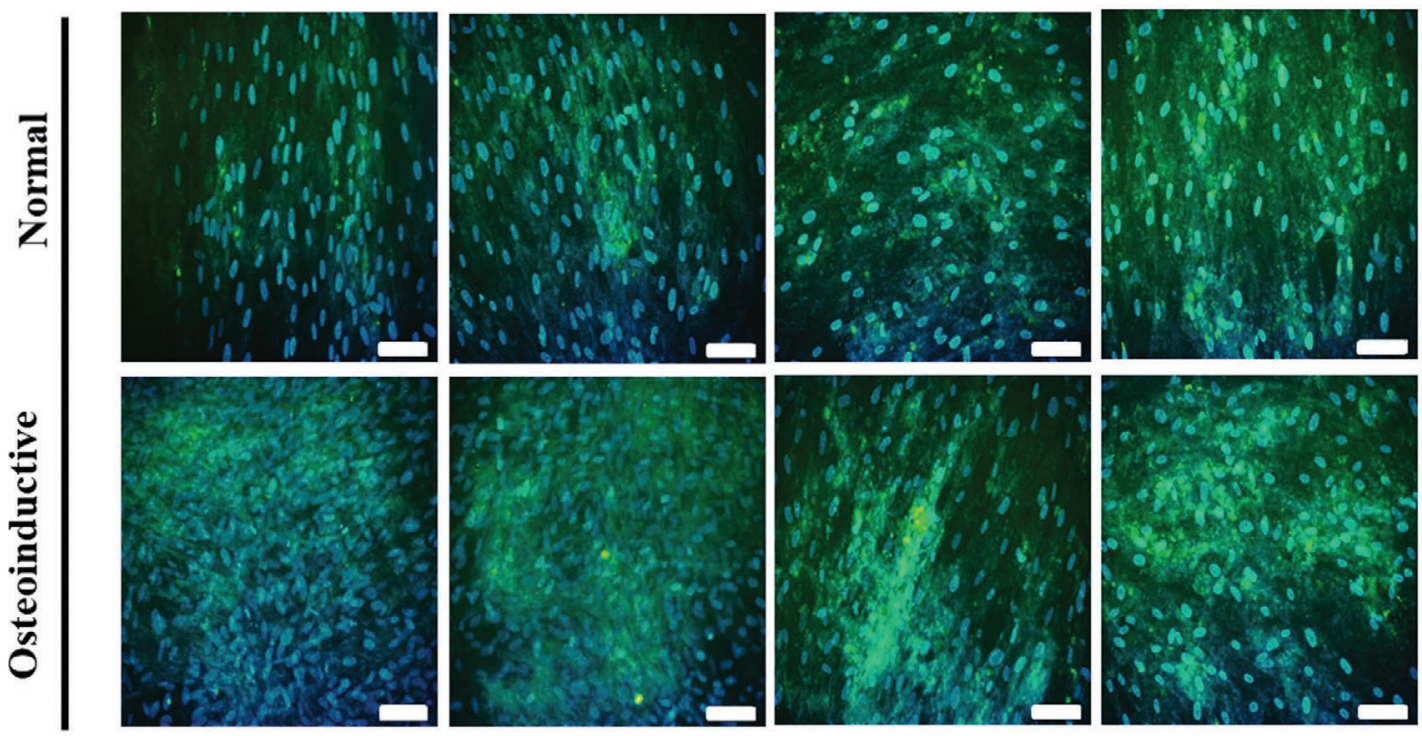

B
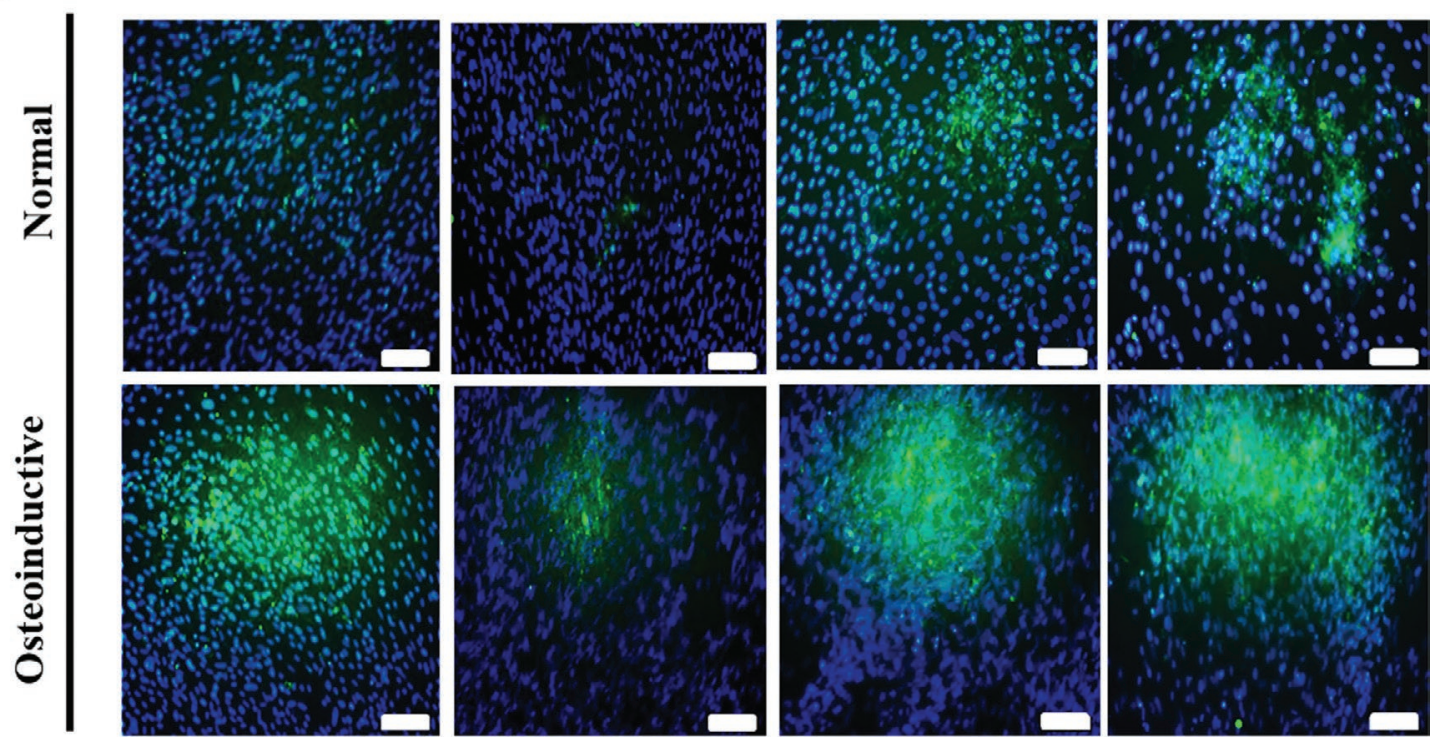

Figure 6. Immunofluorescent staining of bone-specific protein RUNX2 and BSP in ADMSCs at day 21. RUNX2 (A) and BSP (B) proteins were colored green and nuclei were colored blue. Scale bar $=100 \mu \mathrm{m}$.

\subsection{Bone Formation Performance In Vivo}

To evaluate the ability of BGN@GP nanoparticles in improving the reconstruction of calvarial bone defect in vivo, two $3 \mathrm{~mm}$ craniotomy defects were created in SD rats and BGNs/BGN@ GP-contained hydrogel was injected. After treated for 8 and 12 weeks, the bone regeneration performance was evaluated by micro-CT and tissue staining. The analysis of micro-CT showed that more bone-like tissues were formed in the BGN and BGN@GP nanoparticles treated group at 8 and 12 weeks, compared with nanoparticles-free control (Figure 7A). Additionally, as compared to control and BGNs group, BGN@GP significantly enhanced the new bone formation either at
8 or $12 \mathrm{~W}$ (Figure $7 \mathrm{~A}$ ). The calculated new bone regeneration ratio and trabecular thickness further confirmed the efficiency of BGN@GP nanoparticles in improving the bone regeneration (Figure 7B,C), and it was clearly found that the skull defects were nearly repaired completely at 12 weeks with BGN@GP. Subsequently, the histological examination was carried out to further evaluate the bone formation performance. Comparing with blank groups, the H.E. staining indicated that no obvious inflammation for BGNs and BGN@GP treated groups was found in the repaired area (Figure 8A). The Masson staining revealed the significantly increased collagen deposition for BGN@GP group, compared with BGNs and blank group (Figure 8B). H.E. and Masson staining both 
A

Blank
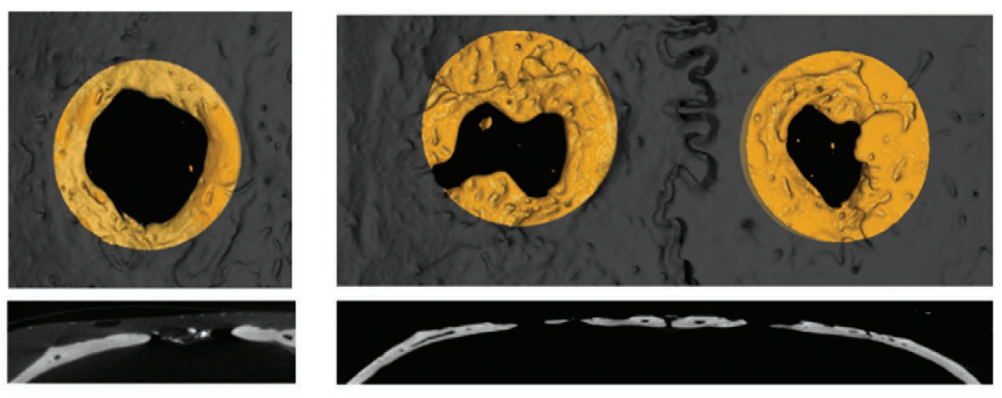

$12 W$
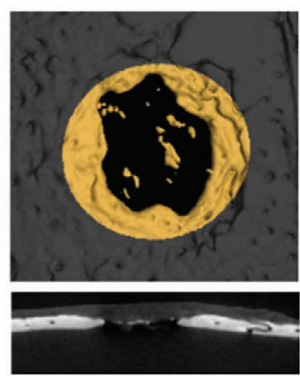

BGN

BGN@GP
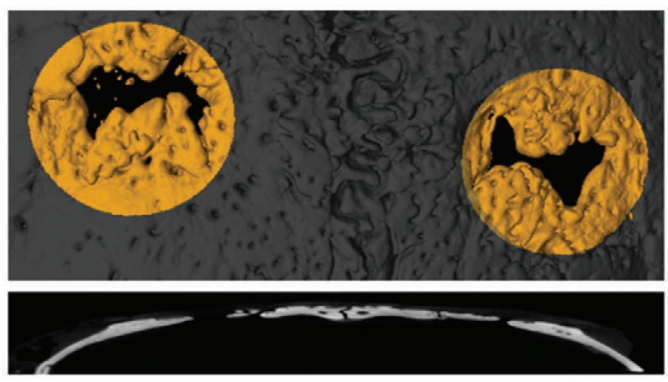

B
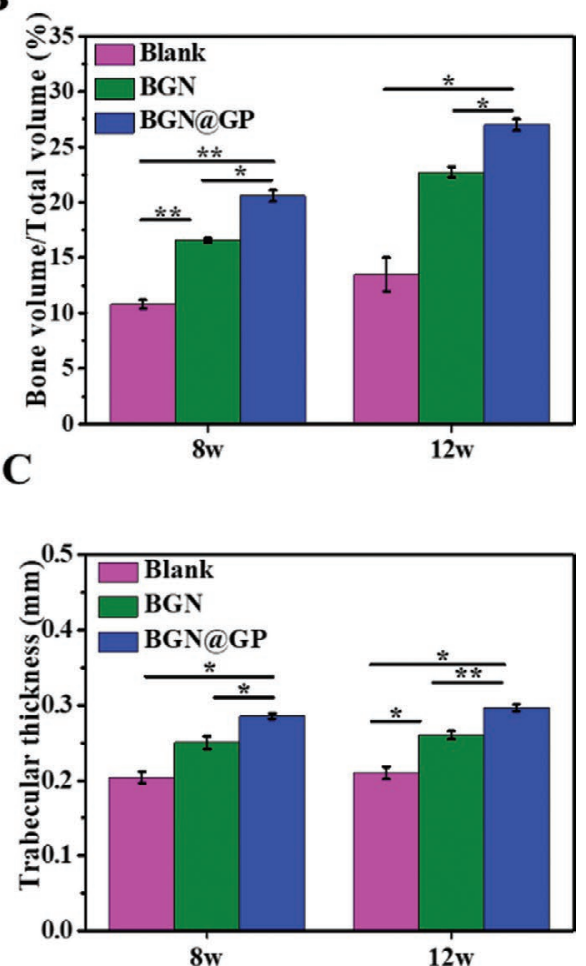

Figure 7. In vivo bone regeneration evaluation. A) Micro-CT images of the bone defects at 8 and 12 weeks after implanting BGNs and BNG@GP. B) New bone formation ratio and C) trabecular thickness of BGN@GP, BGNs and blank groups. $* P<0.05$ and $* * P<0.01$.

showed more regenerated bone tissue formed in the defect area for the BGN@GP group. The results of animal experiment suggested that BGN@GP could significantly enhance the bone regeneration as compared to conventional BGNs.

Monodispersed BGNs possess many special physicochemical and biological properties, however, conventional BGNs showed significant aggregation in physiological condition and low cell uptake and insufficient osteoinductive activity, which limit their wide application in injectable bone regeneration and drug delivery. Here, we demonstrated that the GP decorated BGNs (BGN@GP) showed excellent dispersibility and good cell uptake and enhanced osteogenic differentiation capacity. Compared with conventional BGNs, BGN@GP significantly enhanced the in vitro ALP activity, calcium biomineralization, osteogenic maker genes, and proteins expression of ADMSCs, as well as the in vivo bone formation. The enhanced osteogenic differentiation of ADMSCs was probably attributed to the bioactive feature of BGNs and GP. Previous studies showed that monodispersed BGNs could induce the osteogenic differentiation of ADMSCs through the signaling pathway of TGF-beta. ${ }^{[14,33]}$ The main reasons of GP functionalization enhancing the osteogenic differentiation of BGN on ADMSCs could be shown as follows. First, GP modification significantly enhanced the degradation/ water-dispersion and the cell uptake of BGN (Figure S2, Supporting Information), which would increase the intracellular ions concentration (ICP analysis) and improve the osteogenic differentiation. ${ }^{[38]}$ Compared with BGN, the release concentration of $\mathrm{Si}, \mathrm{Ca}$, and P from BGN@GP significantly increased.
Secondly, $\beta$-glycerophosphate plays an important role in classic osteogenic differentiation protocol for stem cells, ${ }^{[39]}$ as a phosphate source for bone mineral. ${ }^{[40]}$ In our study, the concentration of GP was increased for BGN@GP in normal (2.6 mm) and osteogenic differentiation medium $(12.6 \mathrm{~mm})$, which also contributed to the biomineralization and osteogenic differentiation (Figure 4). Compared to other studies, the current work first demonstrated the effect of BGN@GP in promoting the osteogenic differentiation, calcium biomineralization, and bone regeneration in vivo. This study suggests that BGN@GP may act as promising biomaterials for bioimaging, drug delivery, and bone regenerative medicine.

\section{Conclusion}

In summary, the effect of BGN@GP nanoparticles on the osteogenic differentiation of ADMSCs and in vivo bone regeneration was clarified. BGN@GP showed low cytotoxicity on ADMSCs at low concentration which was similar with BGNs. As compared to BGNs, BGN@GP could significantly enhance the osteogenic differentiation of ADMSCs through stimulating the ALP activity, calcium biomineralization, osteogenic genes (Runx2 and Bsp), and proteins expression (RUNX2 and BSP). The BGN@GP demonstrated better in vivo bone regeneration capacity compared with BGNs. This work provides a facile strategy of biomolecule modification on nanoparticles for enhancing their bone regeneration capacities. 
A

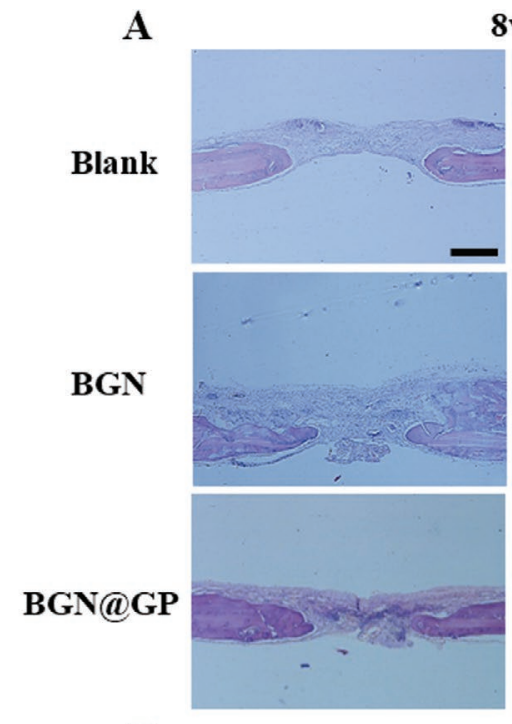

8w
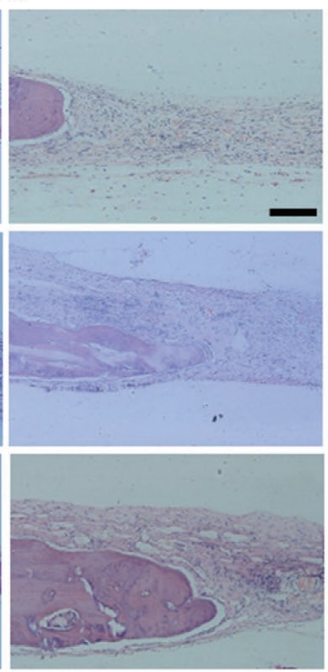

B

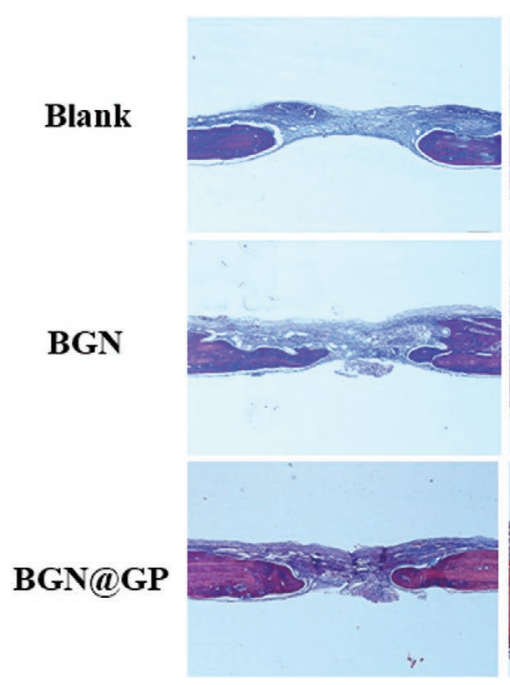

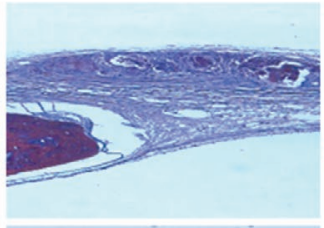
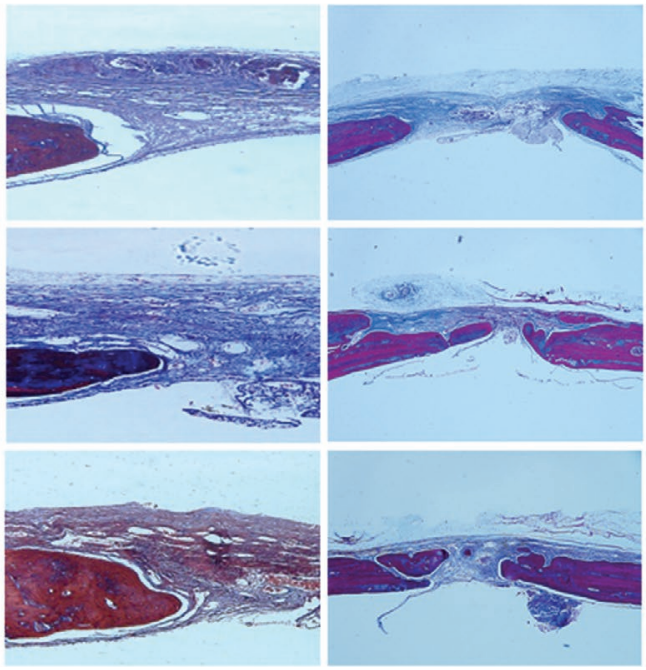

12w
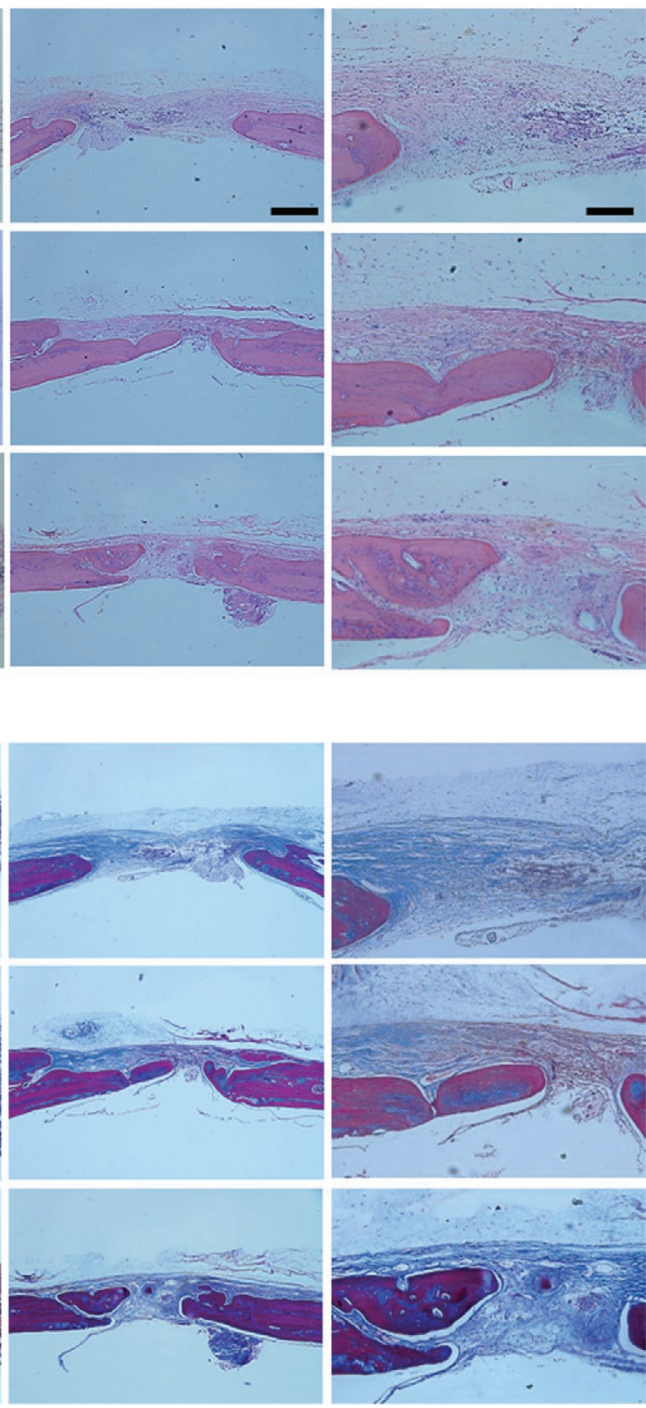

Figure 8. Histological analysis of regenerated bone tissue. A) Representative images of H.E. staining for morphologic evaluation. B) Masson staining for collagen deposition analysis. Scale bar $=50$ and $200 \mu \mathrm{m}$.

\section{Supporting Information}

Supporting Information is available from the Wiley Online Library or from the author.

\section{Acknowledgements}

This work was supported by National Natural Science Foundation of China (Grant No. 51872224), Key Laboratory of Shaanxi Province for Craniofacial Precision Medicine Research, College of Stomatology, Xi'an Jiaotong University (Grant No. 2018LHM-KFKT004), Special Guidance Funds for the Construction of World-class Universities (disciplines), and Characteristic Development in Central Universities (Grant No. PY3A078).

\section{Conflict of Interest}

The authors declare no conflict of interest.

\section{Keywords}

adipose stem cells, bioactive biomaterials, glass nanoparticles, osteogenic differentiation, surface modification

Received: November 25, 2019

Revised: January 12, 2020

Published online: March 3, 2020

[1] R. Langer, D. A. Tirrell, Nature 2004, 428, 487.

[2] A. Khademhosseini, R. Langer, J. Borenstein, J. P. Vacanti, Proc. Natl. Acad. Sci. USA 2006, 103, 2480.

[3] D. Tang, R. S. Tare, L. Y. Yang, D. F. Williams, K. L. Ou, R. O. C. Oreffo, Biomaterials 2016, 83, 363.

[4] G. P. Fadini, S. Ciciliot, M. Albiero, Stem Cells 2017, 35, 106.

[5] S. V. Dorozhkin, Biomaterials 2010, 31, 1465.

[6] D. Marolt, M. Knezevic, G. V. Novakovic, Stem Cell Res. Ther. 2010, $1,10$. 
[7] M. Yu, Y. Du, Y. Han, B. Lei, Adv. Funct. Mater. 2019, 30, 1906013.

[8] W. K. Ramp, K. K. Kaysinger, N. C. Nicholson, J. Bone Miner. Res. 1993, 8, S294.

[9] J. S. Fernandes, P. Gentile, R. A. Pires, R. L. Reis, P. V. Hatton, Acta Biomater. 2017, 59, 2.

[10] A. A. El-Rashidy, J. A. Roether, L. Harhaus, U. Kneser, A. R. Boccaccini, Acta Biomater. 2017, 62, 1.

[11] L. C. Gerhardt, A. R. Boccaccini, Materials 2010, 3, 3867.

[12] C. M. Madl, S. C. Heilshorn, H. M. Blau, Nature 2018, 557, 335.

[13] S. Bose, M. Roy, A. Bandyopadhyay, Trends Biotechnol. 2012, 30, 546.

[14] Y. Guo, Y. M. Xue, W. Niu, M. Chen, M. Wang, P. X. Ma, B. Lei, Part. Part. Syst. Charact. 2018, 35, 1800087.

[15] A. Singh, J. A. Zhan, Z. Y. Ye, J. H. Elisseeff, Adv. Funct. Mater. 2013, 23, 575.

[16] B. Levi, M. T. Longaker, Stem Cells 2011, 29, 576.

[17] Z. T. Chen, C. T. Wu, W. Y. Gu, T. Klein, R. Crawford, Y. Xiao, Biomaterials 2014, 35, 1507

[18] S. Q. Yi, M. Yu, S. Yang, R. J. Miron, Y. F. Zhang, Stem Cells 2017, $35,386$.

[19] Y. L. Zhou, L. Z. Zhang, W. X. Zhao, Y. F. Wu, C. L. Zhu, Y. M. Yang, Biomaterials 2013, 34, 8269.

[20] M. M. Beloti, R. F. Abuna, F. S. de Oliveira, R. B. Kato, A. L. Rosa, FASEB J. 2013, 27, 1146.3.

[21] Z. T. Chen, S. W. Han, M. C. Shi, G. Q. Liu, Z. F. Chen, J. Chang, C. T. Wu, Y. Xiao, Appl. Mater. Today 2018, 10, 184.

[22] A. K. Gaharwar, S. M. Mihaila, A. Swami, A. Patel, S. Sant, R. L. Reis, A. P. Marques, M. E. Gomes, A. Khademhosseini, Adv. Mater. 2013, 25, 3329.

[23] Y. Li, C. Liu, Nanoscale 2017, 9, 4862.

[24] D. D. Dean, Z. Schwartz, L. Bonewald, O. E. Muniz, D. S. Howell, B. D. Boyan, J. Bone Miner. Res. 1993, 8, S241.
[25] M. Chen, F. Zhao, Y. Li, M. Wang, X. Chen, B. Lei, Mater. Sci. Eng., C. 2020, 106, 110153

[26] Y. Xue, W. Niu, M. Wang, M. Chen, Y. Guo, B. Lei. ACS Nano 2020, $14,442$.

[27] L. Shang, K. Nienhaus, G. U. Nienhaus, J. Nanobiotechnol. 2014, 12, 1477.

[28] C. Yang, X. Y. Wang, B. Ma, H. B. Zhu, Z. G. Huan, N. Ma, C. T. Wu, J. Chang, ACS Appl. Mater. Interfaces 2017, 9, 5757.

[29] Q. Chen, R. P. Garcia, J. Munoz, U. P. de Larraya, N. Garmendia, Q. Q. Yao, A. R. Boccaccini, ACS Appl. Mater. Interfaces 2015, 7, 24715.

[30] J. R. Jones, Acta Biomater. 2013, 9, 4457.

[31] Y. Xi, Y. Guo, M. Wang, J. Ge, Y. Liu, W. Niu, M. Chen, Y. Xue, D. D. Winston, W. Dai, B. Lei, C. Lin, Chem. Eng. J. 2020, 383, 123078.

[32] C. T. Wu, Y. H. Zhou, W. Fan, P. P. Han, J. Chang, J. Yuen, M. L. Zhang, Y. Xiao, Biomaterials 2012, 33, 2076

[33] W. Niu, Y. Guo, Y. M. Xue, M. Chen, M. Wang, W. Cheng, B. Lei, Part. Part. Syst. Charact. 2019, 36, 201800087.

[34] Y. M. Xue, Z. J. Zhang, W. Niu, M. Chen, M. Wang, Y. Guo, C. Mao, C. Lin, B. Lei, Part. Part. Syst. Charact. 2019, 36, 201800507.

[35] L. Zhou, Y. W. Xi, Y. M. Xue, M. Wang, Y. L. Liu, Y. Guo, B. Lei, Adv. Funct. Mater. 2019, 29, 1806883.

[36] Q. F. Dang, K. Liu, Z. Z. Zhang, C. S. Liu, X. Liu, Y. Xin, X. Y. Cheng, T. Xu, D. S. Cha, B. Fan, Carbohydr. Polym. 2017, 167, 145.

[37] S. Kim, S. K. Nishimoto, J. D. Bumgardner, W. O. Haggard, M. W. Gaber, Y. Z. Yang, Biomaterials 2010, 31, 4157.

[38] A. Hoppe, N. S. Guldal, A. R. Boccaccini, Biomaterials 2011, 32, 2757.

[39] R. J. Kroeze, M. Knippenberg, M. N. Helder, Adipose deriv. stem cells: methods protoc. 2011, 702, 233

[40] F. Langenbach, J. Handschel, Stem Cell Res. Ther. 2013, 4, scrt328. 